\title{
QT interval and drug therapy (Clinical review from Drug and Therapeutics Bulletin)
}

We made a mistake in Box 2 (Drugs that can cause QT prolongation) of this article (BMJ 2016;353:i2732, doi:10.1136/ bmj.i2732). Under the second heading, "Other cardiac drugs," the list should have only one entry, "Ranolazine." There should then follow the heading, "Antibiotics," followed by the list, "Macrolides (eg erythromycin, clarithromycin, azithromycin), quinolones (eg levofloxacin, moxifloxacin)." 